\title{
Understanding the relationship between intrinsic cardiorespiratory fitness and serum and skeletal muscle metabolomics profile
}

\author{
Alex Castro $^{1 *}$, Renata G. Duft ${ }^{1}$, Lucas M. Silva ${ }^{1}$, Marina L. V. Ferreira ${ }^{1}$, André L. L. Andrade ${ }^{1,2}$,
} Celene F. Bernardes $^{1}$, Cláudia R. Cavaglieri' ${ }^{1}$, Mara P. T. Chacon-Mikahil ${ }^{*}$

\footnotetext{
${ }^{1}$ Laboratory of Exercise Physiology, School of Physical Education, University of Campinas, Campinas, 13083851, São Paulo, Brazil.

${ }^{2}$ School of Medical Sciences, University of Campinas, Campinas, 13083-887, São Paulo, Brazil.

\begin{abstract}
*Corresponding Author:
Alex Castro - Laboratory of Exercise Physiology, School of Physical Education, University of Campinas, Campinas, 13083-851, São Paulo, Brazil; ORCID: https://orcid.org/0000-0002-0298-2672; e-mail: ax.castro@yahoo.com.br; Phone: +55 19 3521-6625.
\end{abstract}

Mara P. T. Chacon-Mikahil - Laboratory of Exercise Physiology, School of Physical Education, University of Campinas, Campinas, 13083-851, São Paulo, Brazil; ORCID: https://orcid.org/0000-0002-6109-5141; e-mail: marapatricia@fef.unicamp.br; Phone: +55 193521-6625.

\section{SUPPORTING INFORMATION}

Figure S1. Representation of the serum $1 \mathrm{D}{ }_{1} \mathrm{H}-\mathrm{NMR}$ spectrum region where the main metabolites were quantified for a single participant. (A): Entire Spectrum; (B): Ornithine; (C): Betaine; (D): Proline; (E): Threonine; (F): Glutamine.

Figure S2. Representation of a skeletal muscle 1D ${ }_{1} \mathrm{H}-\mathrm{NMR}$ spectrum region where the main metabolites were quantified for a single participant. (A): Entire Spectrum; (B): Formate; (C): Fumarate; (D): Lactate; (E): NADP ${ }^{+}$. Table S1. Summary of serum and skeletal muscle pathways related to iCRF. 
A

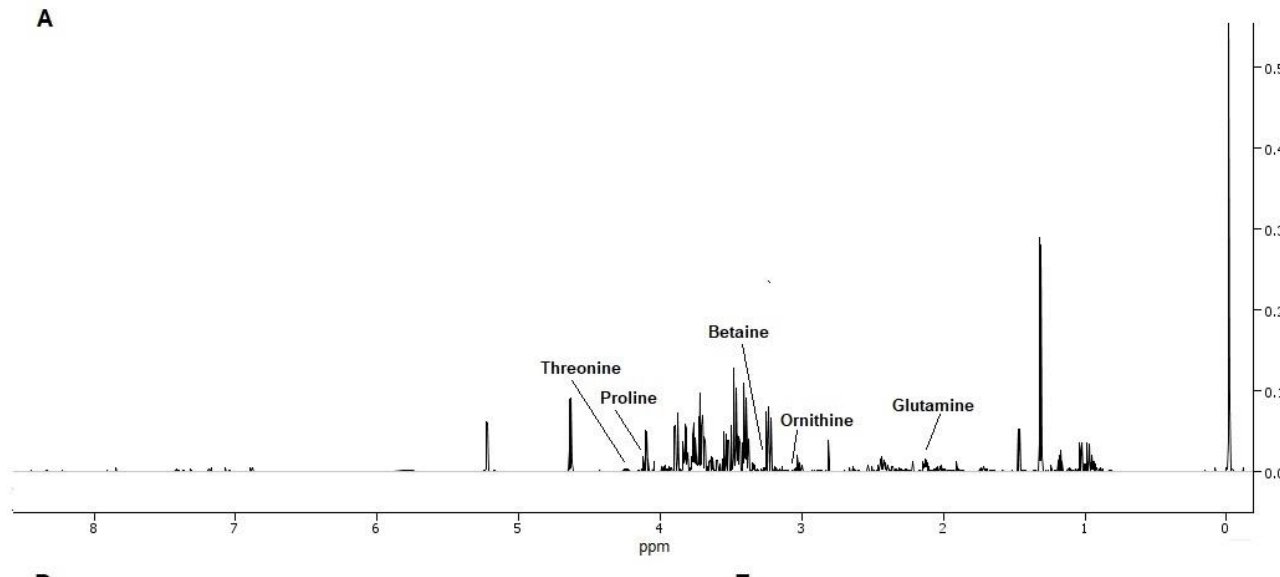

D

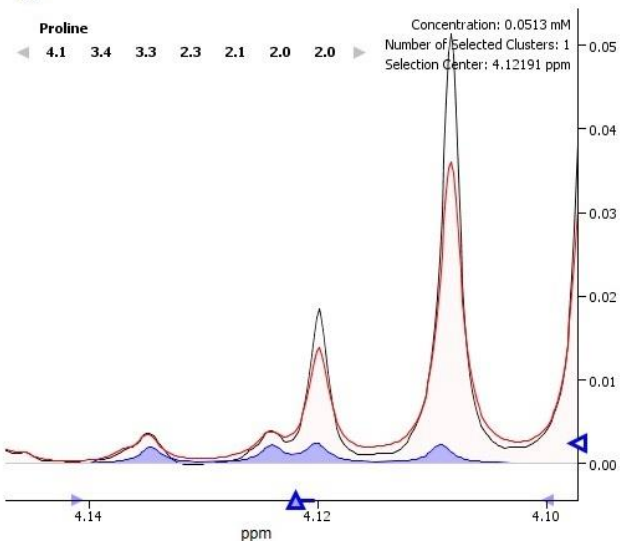

B

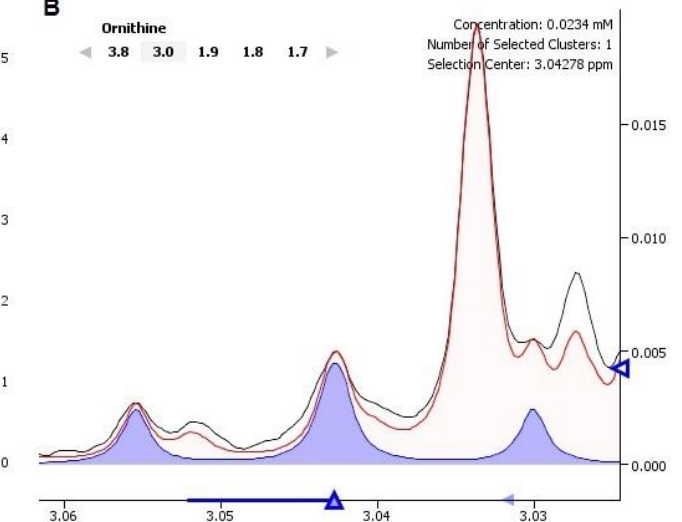

ppm
C Betaine Concentration: $0.0206 \mathrm{~mm}-0.10$

\begin{tabular}{c|c} 
Betaine \\
$4.9 \quad 3.3$ \\
3.9
\end{tabular}

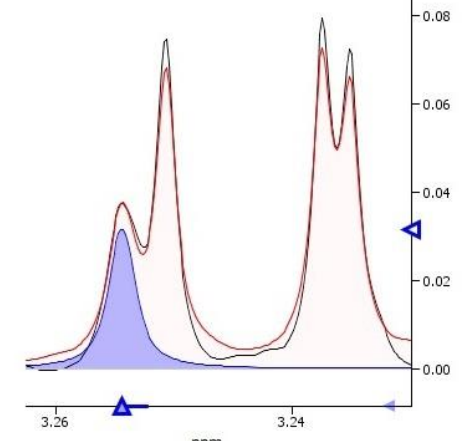

ppm
E

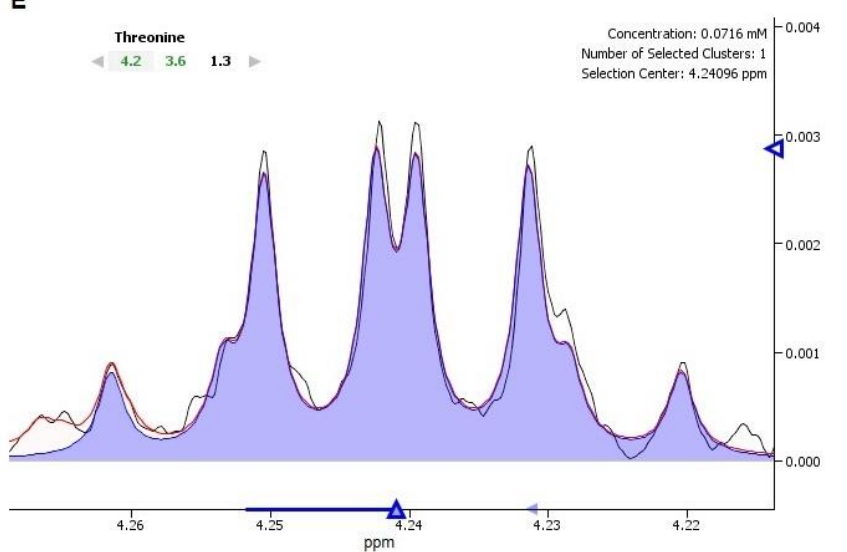

F
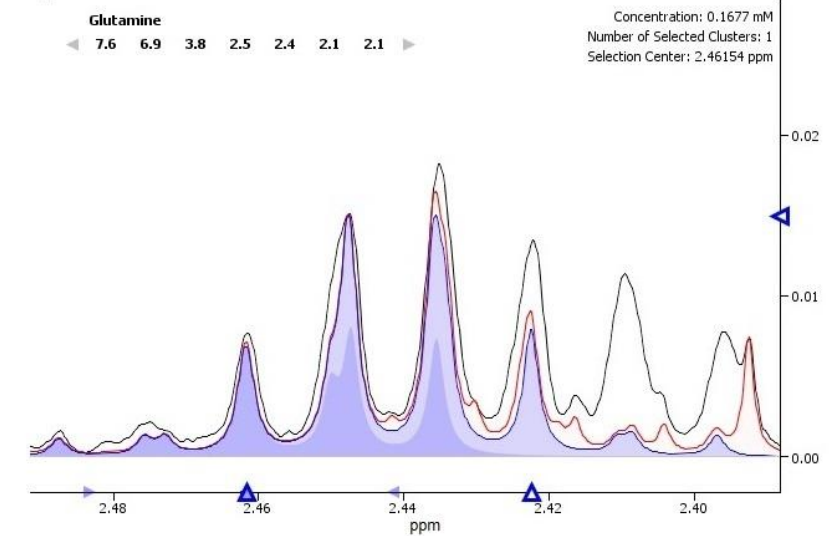

Figure S1. Representation of the serum 1D ${ }_{1} \mathrm{H}-\mathrm{NMR}$ spectrum region where the main metabolites were quantified for a single participant. (A): Entire Spectrum; (B): Ornithine; (C): Betaine; (D): Proline; (E): Threonine; (F): Glutamine. 
A

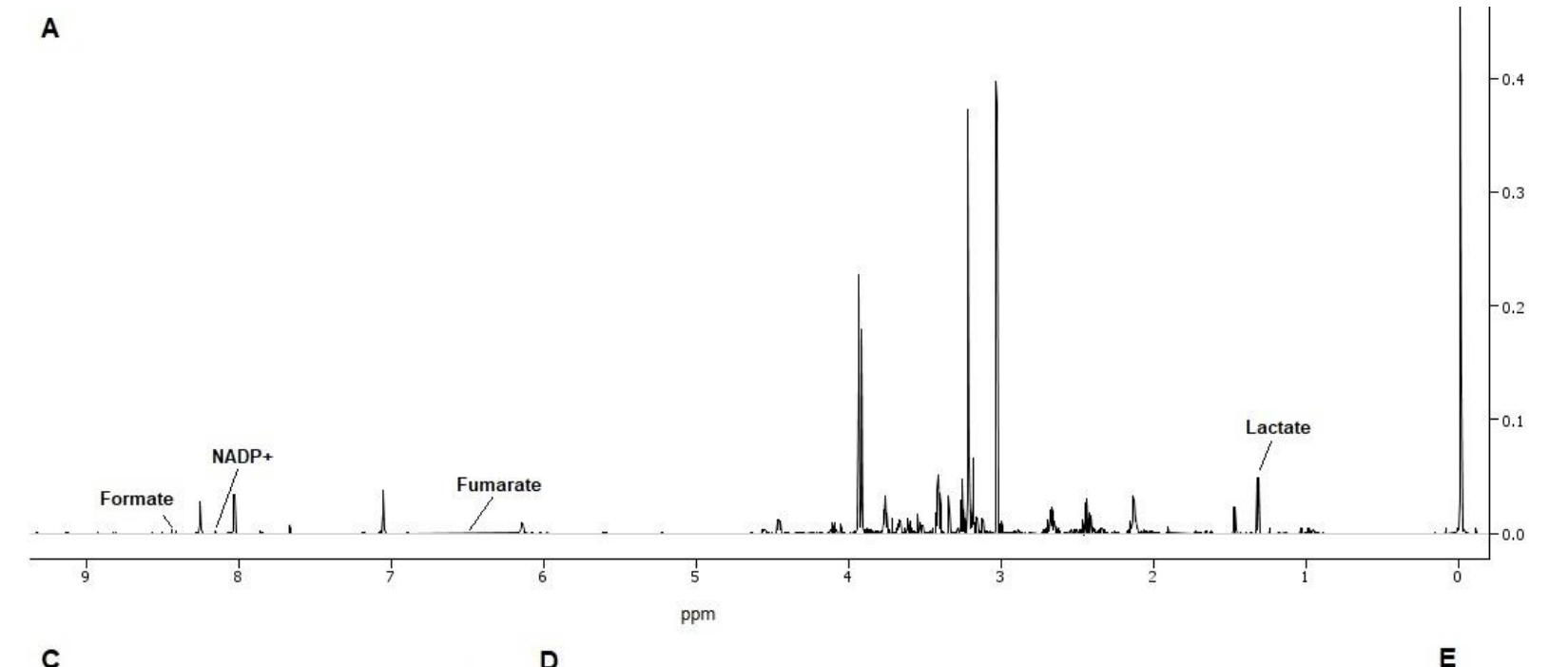

c

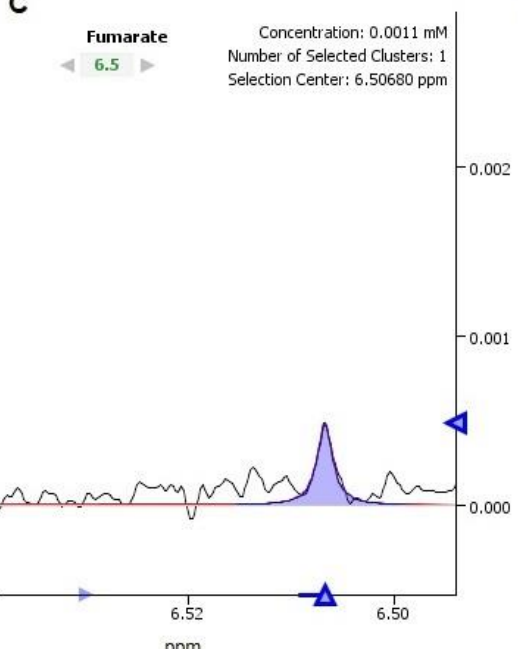

D

ppm

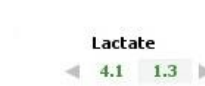

E

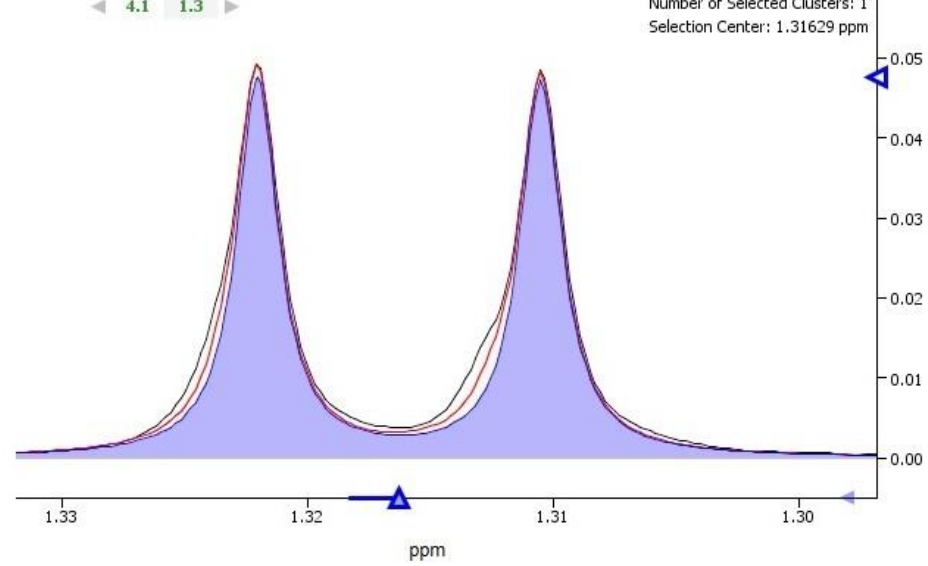

B

$$
\begin{gathered}
\text { Formate } \\
4.4
\end{gathered}
$$

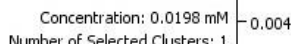
Selection Centert: 8.44414 ppm

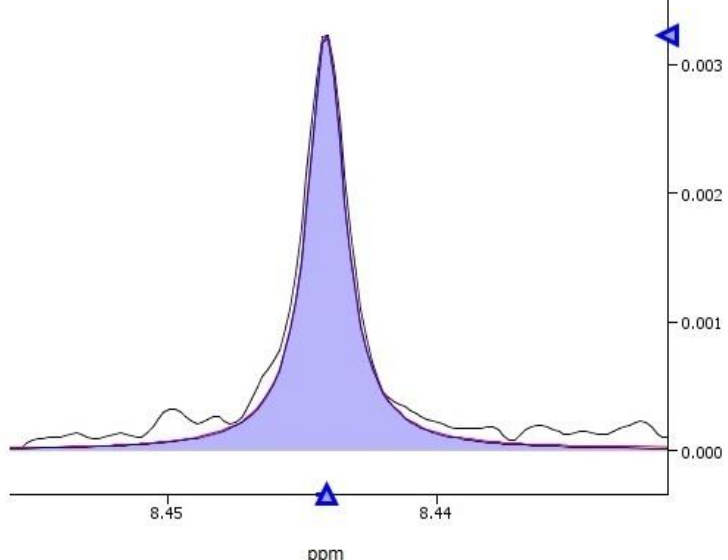

NADP+ $+\quad$ Concentration: $0.0084 \mathrm{~mm}$

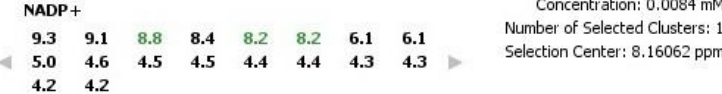

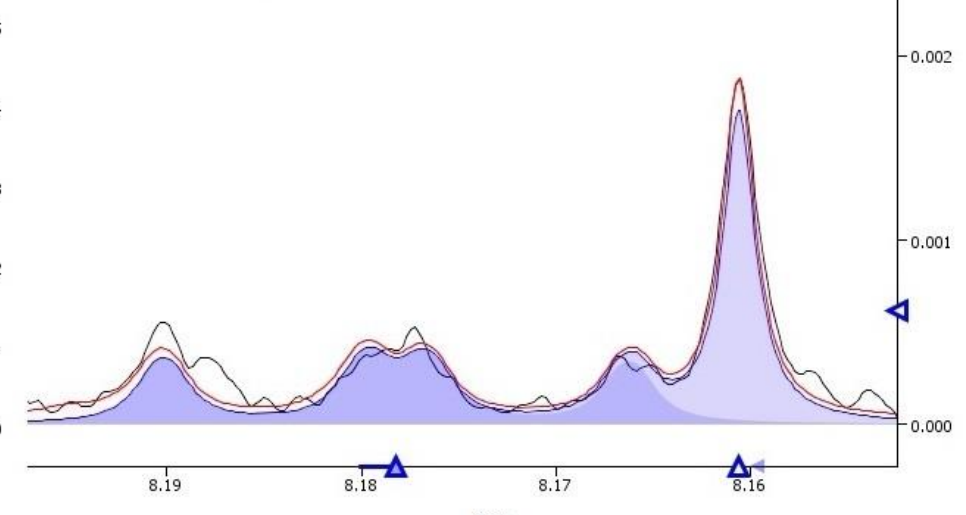

ppm

Figure S2. Representation of a skeletal muscle 1D ${ }_{1} \mathrm{H}-\mathrm{NMR}$ spectrum region where the main metabolites were quantified for a single participant. (A): Entire Spectrum; (B): Formate; (C): Fumarate; (D): Lactate; (E): NADP ${ }^{+}$. 
Table S1. Summary of serum and skeletal muscle pathways related to iCRF.

\begin{tabular}{|c|c|c|c|c|c|c|c|c|c|c|}
\hline $\begin{array}{c}\text { Biologycal } \\
\text { Sample }\end{array}$ & Pathways & Total & Expected & Hits & $\begin{array}{c}\text { Raw } \\
\text { p-values }\end{array}$ & $-\operatorname{LOG}(p)$ & $\begin{array}{l}\text { P-values } \\
\text { Ranking }\end{array}$ & $\begin{array}{c}\text { FDR } \\
\text { (q-values) }\end{array}$ & Impact & Decision \\
\hline Blood & Aminoacyl-tRNA biosynthesis & 48 & 0.3716 & 7 & 0.00000001 & 7.9093 & 1 & 0.0025 & 0.0000 & SIG \\
\hline Blood & Glycine serine and threonine metabolism & 33 & 0.2555 & 4 & 0.00007492 & 4.1254 & 2 & 0.0050 & 0.2961 & SIG \\
\hline Muscle & Pyruvate metabolism & 22 & 0.1419 & 3 & 0.00027962 & 3.5534 & 3 & 0.0075 & 0.1446 & SIG \\
\hline Muscle & Glyoxylate and dicarboxylate metabolism & 32 & 0.2065 & 3 & 0.00087031 & 3.0603 & 4 & 0.0100 & 0.0000 & SIG \\
\hline Blood & Alanine aspartate and glutamate metabolism & 28 & 0.2168 & 3 & 0.00104270 & 2.9818 & 5 & 0.0125 & 0.1138 & SIG \\
\hline Blood & Valine leucine and isoleucine biosynthesis & 8 & 0.0619 & 2 & 0.00150000 & 2.8239 & 6 & 0.0150 & 0.0000 & SIG \\
\hline Muscle & Arginine biosynthesis & 14 & 0.0903 & 2 & 0.00327270 & 2.4851 & 7 & 0.0175 & 0.1168 & SIG \\
\hline Blood & Arginine biosynthesis & 14 & 0.1084 & 2 & 0.00475060 & 2.3233 & 8 & 0.0200 & 0.0609 & SIG \\
\hline Muscle & Glutathione metabolism & 28 & 0.1807 & 2 & 0.01295300 & 1.8876 & 9 & 0.0225 & 0.0215 & SIG \\
\hline Muscle & Alanine aspartate and glutamate metabolism & 28 & 0.1807 & 2 & 0.01295300 & 1.8876 & 10 & 0.0250 & 0.1995 & SIG \\
\hline Blood & Glutathione metabolism & 28 & 0.2168 & 2 & 0.01857800 & 1.7310 & 11 & 0.0275 & 0.0887 & SIG \\
\hline Blood & Glyoxylate and dicarboxylate metabolism & 32 & 0.2477 & 2 & 0.02396100 & 1.6205 & 12 & 0.0300 & 0.1058 & SIG \\
\hline Blood & Arginine and proline metabolism & 38 & 0.2942 & 2 & 0.03309400 & 1.4802 & 13 & 0.0325 & 0.1884 & NS \\
\hline Muscle & Nitrogen metabolism & 6 & 0.0387 & 1 & 0.03815100 & 1.4185 & 14 & 0.0350 & 0.0000 & NS \\
\hline Muscle & D-Glutamine and D-glutamate metabolism & 6 & 0.0387 & 1 & 0.03815100 & 1.4185 & 15 & 0.0375 & 0.5000 & NS \\
\hline Blood & D-Glutamine and D-glutamate metabolism & 6 & 0.0465 & 1 & 0.04563400 & 1.3407 & 16 & 0.0400 & 0.0000 & NS \\
\hline Blood & Nitrogen metabolism & 6 & 0.0465 & 1 & 0.04563400 & 1.3407 & 17 & 0.0425 & 0.0000 & NS \\
\hline Muscle & Taurine and hypotaurine metabolism & 8 & 0.0516 & 1 & 0.05057400 & 1.2961 & 18 & 0.0450 & 0.4286 & NS \\
\hline Muscle & Butanoate metabolism & 15 & 0.0968 & 1 & 0.09292500 & 1.0319 & 19 & 0.0475 & 0.0000 & NS \\
\hline Muscle & Nicotinate and nicotinamide metabolism & 15 & 0.0968 & 1 & 0.09292500 & 1.0319 & 20 & 0.0500 & 0.0000 & NS \\
\hline Muscle & Histidine metabolism & 16 & 0.1032 & 1 & 0.09883400 & 1.0051 & 21 & 0.0525 & 0.0000 & NS \\
\hline Blood & Butanoate metabolism & 15 & 0.1161 & 1 & 0.11052000 & 0.9566 & 22 & 0.0550 & 0.0000 & NS \\
\hline Blood & Histidine metabolism & 16 & 0.1239 & 1 & 0.11747000 & 0.9301 & 23 & 0.0575 & 0.2213 & NS \\
\hline Muscle & Citrate cycle (TCA cycle) & 20 & 0.1290 & 1 & 0.12213000 & 0.9132 & 24 & 0.0600 & 0.0298 & NS \\
\hline Blood & Pantothenate and CoA biosynthesis & 19 & 0.1471 & 1 & 0.13803000 & 0.8600 & 25 & 0.0625 & 0.0000 & NS \\
\hline Blood & Citrate cycle (TCA cycle) & 20 & 0.1548 & 1 & 0.14479000 & 0.8393 & 26 & 0.0650 & 0.0327 & NS \\
\hline
\end{tabular}




\begin{tabular}{|c|c|c|c|c|c|c|c|c|c|c|}
\hline Blood & beta-Alanine metabolism & 21 & 0.1626 & 1 & 0.15150000 & 0.8196 & 27 & 0.0675 & 0.0000 & NS \\
\hline Muscle & Glycolysis/Gluconeogenesis & 26 & 0.1677 & 1 & 0.15605000 & 0.8067 & 28 & 0.0700 & 0.0291 & NS \\
\hline Blood & Propanoate metabolism & 23 & 0.1781 & 1 & 0.16477000 & 0.7831 & 29 & 0.0725 & 0.0000 & NS \\
\hline Muscle & Porphyrin and chlorophyll metabolism & 30 & 0.1936 & 1 & 0.17801000 & 0.7496 & 30 & 0.0750 & 0.0000 & NS \\
\hline Blood & Porphyrin and chlorophyll metabolism & 30 & 0.2323 & 1 & 0.20973000 & 0.6783 & 31 & 0.0775 & 0.0000 & NS \\
\hline Muscle & Arginine and proline metabolism & 38 & 0.2452 & 1 & 0.22038000 & 0.6568 & 32 & 0.0800 & 0.0860 & NS \\
\hline Muscle & Tyrosine metabolism & 42 & 0.2710 & 1 & 0.24082000 & 0.6183 & 33 & 0.0825 & 0.0246 & NS \\
\hline Blood & Glycerophospholipid metabolism & 36 & 0.2787 & 1 & 0.24649000 & 0.6082 & 34 & 0.0850 & 0.0258 & NS \\
\hline Muscle & Primary bile acid biosynthesis & 46 & 0.2968 & 1 & 0.26078000 & 0.5837 & 35 & 0.0875 & 0.0076 & NS \\
\hline Blood & Pyrimidine metabolism & 39 & 0.3019 & 1 & 0.26428000 & 0.5779 & 36 & 0.0900 & 0.0000 & NS \\
\hline Blood & Valine leucine and isoleucine degradation & 40 & 0.3097 & 1 & 0.27012000 & 0.5684 & 37 & 0.0925 & 0.0000 & NS \\
\hline Muscle & Aminoacyl-tRNA biosynthesis & 48 & 0.3097 & 1 & 0.27058000 & 0.5677 & 38 & 0.0950 & 0.0000 & NS \\
\hline Blood & Primary bile acid biosynthesis & 46 & 0.3561 & 1 & 0.30429000 & 0.5167 & 39 & 0.0975 & 0.0076 & NS \\
\hline Blood & Purine metabolism & 65 & 0.5032 & 1 & 0.40307000 & 0.3946 & 40 & 0.1000 & 0.0000 & NS \\
\hline
\end{tabular}

FDR: false discovery rate of 0.1 . All identified pathways were ranked based on their p-values from smallest to largest. Then, the FDR values were calculated for each pathway (q), assuming $\mathrm{q}=\left(0.1^{\times} i\right) / m$, where $i$ denotes the $i$-th position of the pathway in the ranking of p-values (in the $i$-th row) and $m$ the total number of ranked pathways. When the p-values of the pathways were less than the q-values, the pathway was declared to be significantly enriched; SIG: Significant pathway at the FDR level; NS: Non-significant pathway at the FRD level. 